\title{
First-Catch, Mid-Stream and Catheterised urine: A Comparative Study of Male Urinary Microbiome by Expanded Quantitative Urine Culture and Next- Generation Sequencing
}

Jan Hrbacek ( $\square$ honzahrbacek@gmail.com )

Thomayerova Nemocnice https://orcid.org/0000-0002-4243-8698

\section{Daniel Morais}

Institute of Microbiology Czech Academy of Sciences: Mikrobiologicky ustav Akademie ved Ceske republiky

\section{Pavel Cermak}

Fakultní Thomayerova nemocnice: Thomayerova nemocnice

Vitezslav Hanacek

Fakultní Thomayerova nemocnice: Thomayerova nemocnice

Roman Zachoval

Fakultní Thomayerova nemocnice: Thomayerova nemocnice

\section{Research}

Keywords: urinary tract, males, microbiota, expanded quantitative urine culture, next-generation sequencing

Posted Date: July 15th, 2021

DOI: https://doi.org/10.21203/rs.3.rs-700720/v1

License: (c) (1) This work is licensed under a Creative Commons Attribution 4.0 International License. Read Full License 


\section{Abstract}

\section{Background}

Numerous studies have emerged in the past decade investigating human urinary microbiota. Alterations in the microbial composition of urine have been linked to structural and functional abnormalities of the lower urinary tract. There has been considerable variation in the methodology of the studies published so far including the cornerstone of any biomedical analysis: sample collection. The aim of this study was to describe the urinary microbiota of first-catch voided urine (FCU), mid-stream voided urine (MSU) and aseptically catheterised urine in men and find the most suitable approach to urine sample collection for the purpose of male urinary microbiota investigations.

Results

Forty-nine men (mean age 71.3 years) undergoing endoscopic procedures in our Department of Urology were enrolled in the study. Each of them contributed three samples: first-catch urine (FCU), mid-stream urine (MSU) and a catheterised urine sample. The samples were subjected to next-generation sequencing (NGS, $n=35$ ) and expanded quantitative urine culture (EQUC, $n=31$ ).

Using NGS, Bacteroidetes, Firmicutes, and Proteobacteria were the most abundant phyla in our population. The most abundant genera (in order of relative abundance) included: Prevotella, Veillonella, Streptococcus, Porphyromonas, Campylobacter, Pseudomonas, Staphylococcus, Ezakiella, Escherichia and Dialister. Eighty-two of 105 samples were dominated by a single genus. FCU, MSU and catheterised urine samples differed significantly using ANOVA in three out of five alpha-diversity measures $(p<0.05)$ : estimated number of operational taxonomic units, Chao1 and abundance-based coverage estimators. There were no differences found in Simpson and Shannon indices. Beta-diversity comparisons using the PIME method (Prevalence Interval for Microbiome Evaluation) resulted in clear clustering of urine samples according to the mode of sampling.

EQUC detected cultivable bacteria in 30/31 (97\%) FCU and 27/31 (87\%) MSU samples. Only 4/31 (13\%) of catheterised urine samples showed bacterial growth.

Conclusions

Urine samples obtained by transurethral catheterisation under aseptic conditions differ from spontaneously voided urine samples and represent a better reflection of urinary bladder microbiota. Catheterised urine is the most appropriate way to sample urine in future studies of urinary bladder pathological conditions and their relation to the urinary microbiota.

\section{Background}

The human urinary tract had traditionally been considered a sterile environment unlike other body niches such as the gut, oropharynx or vagina; hence, it was not included in the Human Microbiome Project [1]. 
However, the last decade has brought evidence of microbial communities residing in the female [2], [3] and male [4], [5] urinary tract. Various microorganisms have been cultured from urine using expanded quantitative urinary culture (EQUC) and much more prokaryotic diversity unearthed using next-generation sequencing (NGS) [6].

Female urinary microbiota has been given a broad attention; it was less so in case of male urinary microbiota (MUM). And while the microbiota of the female urinary bladder overlaps with that of the vagina to the point that a term "female urogenital microbiota" has been suggested [7], little is known about potential differences in the microbiota along the male urinary tract. The bacterial genera detected in first-catch urine (FCU) are also present in urethral swab specimens; Lactobacillus, Sneathia, Veillonella, Corynebacterium and Prevotella were the most abundant ones according to a study from 2011 [4]. Anecdotic evidence has suggested certain differences between microbial compositions of voided and catheterised urine samples in males [8]-[10]. Differences in beta- (but not alpha-) diversity were reported in a study of urinary bladder cancer patients between voided and cystoscopy-obtained urine samples [8]. Another study reported that urethra and bladder microbiomes did not differ in their taxonomic composition but rather in taxonomic structure (relative abundances of several genera) [9]. And while the prevalence of bacteria in catheterised urine correlated with the degree of lower urinary tract symptoms in men with enlarged prostates, this relationship was not demonstrated on voided samples [10].

Alterations of the urinary microbiome have been associated with functional [11]-[13] and anatomical [10] abnormalities of the urinary tract and with the presence of genitourinary malignant disease [14], [15]. Urge urinary incontinence was associated with higher microbiota diversity [11], [12] and increased diversity also correlated with poor response to anticholinergic (for bladder overactivity) treatment [11]. Changes in the urinary microbiota were reported in patients after spinal cord injury [13] and even among patients with chronic kidney disease where lower diversity was associated with more advanced renal insufficiency [12]. Patients with bladder cancer were reported to have higher microbiota richness compared to healthy controls [14]. Chronic pelvic pain syndrome was associated with a greater alpha-diversity and greater prevalence of anaerobic bacteria than urine of controls without the condition [16]. Conversely, women with intersticial cystitis/painful bladder syndrome had less diverse microbiota than controls [17].

Despite studies looking for and finding differences in composition of the urinary microbiota in a variety of clinical scenarios, an elementary methodological question - i.e. how to collect urine samples to ensure an exact reflection of the urinary bladder microbiota and comparability of future studies - remains unresolved.

The aim of the present study was to describe male urinary microbiota of first-catch voided urine (FCU), mid-stream voided urine (MSU) and aseptically catheterised urine in subjects without symptoms of a urinary tract infection (UTI) and with a negative standard urine culture result. Secondly, we aimed to identify the most suitable sample collection method for the characterization of bacterial communities residing in the male urinary tract. 


\section{Methods}

This is a prospective observational study. Its objective is to investigate within-subject diversity of MUM obtained by three different methods of urine sampling and to define the most suitable approach to the study of the microbiota residing in the male urinary bladder.

\section{Population}

The study participants were recruited among patients undergoing endoscopic procedures for benign or malignant conditions of the urinary tract in the Department of Urology, 3rd Medical Faculty of Charles University and Thomayer Hospital, Prague, Czech Republic. To be included in the study, patients had to have a negative result of standard urine culture preoperatively, no foreign body in the urinary bladder (such as indwelling catheters, ureteric stents or bladder stones) and not have used antibiotic treatment for any medical condition in the past 6 weeks. The study was approved by the institutional review board (ethics committee) and informed consent was obtained from all all participants prior to enrollment.

\section{Urine sampling}

Participants were instructed on proper urine sample collection and were asked to provide an FCU and MSU specimens in two separate sterile containers in the morning before surgery. A third specimen was obtained in theatre at the beginning of the procedure after desinfection of the genital area, surgical draping and immediately upon endoscope insertion. A water-based jelly not containing chlorhexidine or any other desinfectant (Optilube, Optimum Medical Solutions, Leeds, UK) was used to minimize the chances of harm to any presumed living intravesical microorganisms. All samples were stored at $4^{\circ} \mathrm{C}$ before inoculation and processed on the same day with EQUC or frozen and stored at $-20^{\circ} \mathrm{C}$ until DNA extraction.

\section{Expanded quantitative urine culture}

Only subjects with "no growth" reported on routine preoperative culture were eligible for the EQUC part of the study. Patients with preoperative cultures deemed negative for clinical purposes but reported as "commensal flora" or "suspected contamination" were excluded. Antibiotic prophylaxis, where indicated, was administered after urine samples were obtained. EQUC protocol was based on previous description by Hilt et al. [6]. A $100 \mu \mathrm{L}$ aliquot of each urine sample was inoculated with a sterile plastic loop onto agar plates ( $90 \mathrm{~mm}$ in diameter) and in liquid broth. EQUC culture conditions were as follows: 1. Columbia blood agar (CBA) incubated at $37^{\circ} \mathrm{C}$ for 48 hours; 2 . CBA incubated at $30^{\circ} \mathrm{C}$ for 48 hours; 3 . CBA incubated at $37^{\circ} \mathrm{C}$ in a $5 \% \mathrm{CO}_{2}$ incubator for 48 hours; 4 . chocolate agar incubated at $37^{\circ} \mathrm{C}$ in a $5 \% \mathrm{CO}_{2}$ incubator for 48 hours; 5 . and 6 . Schaedler blood agar at $37^{\circ} \mathrm{C}$ in a Campy gas mixture $\left(5 \% \mathrm{O}_{2}, 10 \% \mathrm{CO}_{2}\right.$ and $85 \% \mathrm{~N}$ ) in Oxoid Anaerojar; 7. thioglycolate broth incubated at $37^{\circ} \mathrm{C}$ for 5 days, then inoculated on $\mathrm{CBA}$ and incubated at $37^{\circ} \mathrm{C}$ for another 48 hours. CBA and Schaedler agar were prepared in the laboratory from dried base (Bio-Rad, Berkeley, CA, USA). $5 \%$ sheep blood (LMS - Labmediaservis, Czech Republic) 
was added into the medium at $38^{\circ} \mathrm{C}$. Thioglycolate broth was prepared from dehydrated powder (Bio-Rad, Berkeley, CA, USA). Chocolate agar was obtained as commercial product (Bio-Rad, Berkeley, CA, USA).

The number of colonies was then counted on agar plates with inoculated urine. Growth was visually detected in the thioglycolate broth and colonies were counted on CBA after inoculation and incubation.

Matrix assisted laser desorption ionization-time of flight mass spectrometry (MALDI-TOF, Bruker Daltonik GmbH, Leipzig, Germany) was used for the identification of individual bacteria.

\section{DNA extraction and PCR}

Bacterial 16S rRNA gene was extracted from urine samples using Eligene Urine Isolation Kit (Elisabeth Pharmacon, Brno, Czech Republic) according to manufacturer's instructions. Briefly, urine was vortexed for 15 seconds, centrifuged at $6000 \mathrm{xg}$ for 20 minutes, the supernatant was discarded and and pellet resuspended in $200 \mu \mathrm{l}$ of molecular grade water, $200 \mu \mathrm{l}$ of MI3 solution, and $20 \mu \mathrm{l}$ of Proteinase $\mathrm{K}$ was added. After 15 second vortexing, the mixture was incubated for 15 minutes at $65^{\circ} \mathrm{C}$. The lysate was cetrifuged at $6000 \mathrm{xg}$ for 5 minutes. The supernatant was transferred to microtube and $210 \mu \mathrm{l}$ of Ml4 solution added. The lysate was centrifuged for 1 minute at $13,000 \mathrm{xg}$.

The primers 515F (5- GTGCCAGCMGCCGCGGTAA) and 806R (5- GGACTACHVGGGTWTCTAAT) were used to amplify the hypervariable region V4 of the 16S rRNA gene. Each forward primer was barcoded by a sequence nucleotides designed to multiplexing of different samples [18]. PCR was performed in triplicates, and every reaction contained $5 \mu \mathrm{L}$ of $5 \times \mathrm{X} 5$ Reaction Buffer for Q5 High-Fidelity DNA polymerase; $0.25 \mu \mathrm{L}$ Q5 High-Fidelity DNA polymerase; $5 \mu \mathrm{L}$ of $5 \times \mathrm{XQ}$ HighGC Enhancer; $1.5 \mu \mathrm{L}$ ofBSA $(10 \mathrm{mg} / \mathrm{mL}) ; 0.5 \mu \mathrm{L}$ of PCR Nucleotide Mix $(10 \mathrm{mM}) ; 1 \mu \mathrm{L}$ of primer $515 \mathrm{~F}(10 \mu \mathrm{M}) ; 1 \mu \mathrm{L}$ of primer $806 \mathrm{R}$ $(10 \mu \mathrm{M}) ; 1.0 \mu \mathrm{L}$ of template DNA and sterile ddH2O up to $25 \mu \mathrm{L}$. Conditions for amplification started at $94^{\circ} \mathrm{C}$ for 4 min followed by 25 cycles of $94^{\circ} \mathrm{C}$ for $45 \mathrm{~s}, 50^{\circ} \mathrm{C}$ for $60 \mathrm{~s}, 72^{\circ} \mathrm{C}$ for $75 \mathrm{~s}$ and finished with a final setting of $72^{\circ} \mathrm{C}$ for $10 \mathrm{~min}$.

Statistical analyses

Demographic and clinical data were analysed as continuous or categorical variables and reported as median and inter-quartile range (IQR) or percentages as appropriate. Bacterial growth using EQUC was assessed as present or absent and correlation between the three samples from individual patients were evaluated. XLSTAT (Addinsoft, New York, USA) was used for statistical calculations.

The sequencing data were processed using SEED 2.1.2 [19]. Pair-end reads were merged using fastq-join [20]. Sequences with ambiguous bases were omitted as well as sequences with average quality PHRED score $<30$. The chimeric sequences were detected and removed using Usearch 7.0.1090, and clustered into OTUs using the uparse agorithm [21] at a $97 \%$ similarity level. The most abundant sequence from each cluster [22] was assigned to the closest hits at the genus level using the RDP Naïve Bayesian Classifier Version 2.11 method [23] for bacteria. Sequences identified as non-bacterial were discarded. The DNA sequences have been deposited at the NCBI SRA under the accession number PRJNA744742. 
Analyses of alpha- and beta-diversity were perfomed using the packages Vegan [24], phyloseq [25] and Prevalence Interval for Microbiome Evaluation (PIME) [26] from R language [27]. The PIME algorithm is used to identify the most relevant OTUs to separate treatments or groups of samples. The method is based on the concept of prevalence, considering that high abundance OTUs that have low prevalence among samples from the same treatment/group are not relevant to characterise a treatment/group of samples. It uses Random Forests to classify the groups of samples using high prevalence OTUs.

\section{Results}

A total of 49 men (147 urine samples) were included in the study between August 2019 and February 2021 in whom all three urine samples and full clinical information were available. EQUC and NGS were performed on urine samples from 31 and 35 subjects, respectively. Median age of participants was 71.3 years (IQR 63-76). Thirty-one men were undergoing endoscopic urological procedures for urinary bladder cancer while 18 had a benign condition (benign prostate hyperplasia [ $=9]$ or upper urinary tract stone disease $[n=9]$ ). Standard urine culture with a negative result was performed as part of preoperative assessment 9 days (median; IQR 4-14) before procedure.

Details on clinical and demographic data of the study population are provided in Table 1. 
Table 1

Clinical and demographic variables of the study population $(n=49)$

\begin{tabular}{|ll|}
\hline Variable & \\
\hline Age, mean (IQR) & $71.3(63-76)$ \\
\hline Ethnicity: Caucasian, $\mathrm{n}(\%)$ & $49(100 \%)$ \\
\hline BMI, mean (IQR) & $28.7(26-32)$ \\
\hline Hypertension, $\mathrm{n}(\%)$ & $37(76 \%)$ \\
\hline Diabetes, $\mathrm{n}(\%)$ & $17(35 \%)$ \\
\hline Hyperlipidemia, $\mathrm{n}(\%)$ & $23(47 \%)$ \\
\hline Chronic kidney disease ${ }^{1}, \mathrm{n}(\%)$ & $10(20 \%)$ \\
\hline Bladder cancer, $\mathrm{n}(\%)$ & $31(63 \%)$ \\
\hline History of instillation treatment for bladder cancer ${ }^{2}, \mathrm{n}(\%)$ & $4(8 \%)$ \\
\hline History of pelvic radiotherapy, $\mathrm{n}(\%)$ & $3(6 \%)$ \\
\hline Current smoker, $\mathrm{n}(\%)$ & $11(22 \%)$ \\
\hline Post-void residual urine ${ }^{3}, \mathrm{n}(\%)$ & $8(16 \%)$ \\
\hline IQR - interquartile range, BMI - body mass index & \\
\hline 1 estimated glomerular filtration rate (CKD-EPI eGFR) < 60 ml/min. & \\
\hline 2 mitomycin-C, epirubicin or Bacillus Calmette-Guérin & \\
\hline 3 40 mL or more left in the bladder after spontaneous voiding as measured by ultrasound \\
\hline
\end{tabular}

NGS

Triplets of urine samples from 38 men were subjected to NGS. Three were excluded, as some of their samples did not provide a sufficient amount of DNA sequences leaving 35 subjects for analysis.

A total of 2208522 raw reads were obtained from 105 urine samples with a median number of 16674 reads per sample. After filtering out low quality sequences, removing chimeras and non bacterial DNA we were left with 732779 reads and a median number of 3166 reads per sample. After exclusion of singletons and doubletons, the reads were assigned to 53 phyla, 107 classes, 207 orders, 400 families and 995 genera.

The phyla Bacteroidetes, Firmicutes, and Proteobacteria represented the majority of sequences in the three types of urine specimens with differing relative abundances. The 10 most abundant genera (in order of relative abundance) were: Prevotella, Veillonella, Streptococcus, Porphyromonas, Campylobacter, 
Pseudomonas, Staphylococcus, Ezakiella, Escherichia and Dialister representing around 530.000 sequences, approximately $72 \%$ of the total sequences obtained after removing errors and contaminants. Figure 1 depicts the relative abundances at genus level in all samples.

FCU, MSU and catheterised urine samples differed significantly using ANOVA in three out of five alpha diversity measures used: estimated number of OTUs $(p<0.05)$, Chao1 $(p<0.05)$ and abundance-based coverage estimators (ACE) index $(p<0.05)$; there were no statistically significant differences in Simpson and Shannon indices (Table 2 and Fig. 2).

Table 2

Alpha diversity measures for first-catch, mid-stream and catheterised urine.

\begin{tabular}{|lcccl|}
\hline & FCU & MSU & catheterised & p \\
\hline $\begin{array}{l}\text { Observed } \\
\text { genera }\end{array}$ & 171.3 & 157.8 & 103.9 & 0.0002 \\
\hline Chao1 & 285.1 & 280.0 & 160.6 & $<0.0001$ \\
\hline ACE & 315.6 & 307.3 & 168.3 & $<0.0001$ \\
\hline Shannon & 2.62 & 2.61 & 2.49 & 0.66 \\
\hline Simpson & 0.76 & 0.79 & 0.80 & 0.50 \\
\hline
\end{tabular}

Beta-diversity comparisons using the PIME method (removing noisy OTUs from samples and keeping only the OTUs that are adding biological meaning to the group of samples) resulted in clear clustering of urine samples according to the mode of sampling (Fig. 3).

Regardless of whether urine samples originated from FCU, MSU or catheterisation, they were mostly dominated by a single genus (82/105 samples, $78.1 \%)$, less commonly two $(21 / 105,20.0 \%)$ or three genera $(2 / 105,1.9 \%)$ were evenly represented. Prevotella, Porphyromonas and Pseudomonas were the most frequently found genera among the top-three in the FCU and MSU samples while Pseudomonas, Ferroglobus and Prevotella featured most often amont the top-three in catheterised urine specimens.

\section{EQUC}

Among 31 FCU samples subjected to EQUC, 30 (97\%) showed presence of cultivable bacteria. MSU specimens yielded a positive EQUC result in $27(87 \%)$ cases. Only four of $31(13 \%)$ specimens obtained via transurethral instrumentation were EQUC-positive.

A total of 23 individual species and 13 genera were identified using EQUC with a median of 2 (range 1-4) isolates per urine sample (of those with a positive result). Enterococcus faecalis and Staphylococcus epidermidis were the most prevalent (10 of 31 each, 32 \%) among both FCU and MSU samples, 
respectively. Corynebacterium glucuronolyticum was the third most frequent microorganism isolated (8/31 [26 \%] and 7/31 [23\%] in FCU and MSU, respectively). Among specimens drawn under aseptic conditions from the urinary bladder during theatre procedures, only three yielded a positive EQUC result: C. glucuronolyticum in one, E. faecalis in two and S. epidermidis in one patient. All of these four microorganisms were also present in the particular subject's FCU and MSU samples.

In terms of concordance between FCU and MSU samples, both showed identical EQUC species-level result in 21 cases (68\%). In the remaining 10 patients, the results were discordant as follows: a positive firstcatch with a sterile mid-stream sample $(n=4)$; same number of detected isolates but different specieslevel composition $(n=3)$; and different number of isolates and their composition $(n=3)$. Supplementary table S1 (Additional file 1) shows a complete list of all subjects and their EQUC findings.

A total of 18 patients had their triplets of samples subjected to both EQUC and NGS. Out of 77 EQUCdetected isolates in these 54 samples, 61 microorganisms (79\%) were also detectable by NGS.

\section{Discussion}

This is the only study to date dedicated specifically to the investigation of the potential differences among FCU, MSU and catheterised urine in men. We proved that catheterised urine samples differ in microbiota composition from spontaneously voided urine. This finding has important consequences for future MUM studies.

Dong et al. reported on the microbial communities of FCU samples as early as 2011 [4]. The genera detected in his population of men from an STD clinic were also detected among our first-catch samples and some of them also belonged to the most abundant OTUs: Prevotella, Streptococcus, Veillonella and Staphylococcus, to name the first four. On the contrary, Sneathia spp. was not detected in our FCU samples at all. Different age of subjects in the Dong study (28 years), different DNA analysis method (pyrosequencing) or the variable region of the 16s rRNA gene (V1-V3) might all explain this difference but there is a substantial degree of concordance between our and Dong et al. data.

Bajic et al. were the first to note that MSU differs from catheterised samples from the same individual in their study of male lower urinary tract symptoms and suggested that catheterisation was the most appropriate method for sampling the male bladder microbiota. In a study of lower urinary tract symptoms, men with benign prostate hyperplasia were assessed for symptom severity; increase in the symptom score was associated with higher odds of detectable bacteria in catheterised urine, although no specific genera were associated with the degree of symptoms; no such association was observed when analyzing spontaneously voided urine [10]. Recently, Hourigan et al. reported significant differences in beta-diversity (but not alpha-diversity) measures between voided and catheterised urine ( $n=14 \mathrm{men})$. The sample was too small to detect any particular OTU to be enriched in voided or catheterised samples, respectively [8]. Pohl et al. examined MSU and catheterised urine from 14 males of four different ethnic origins and reported differences in relative abunance of several OTUs between voided and catheterised urine [9]. 
In the present study, we detected a significant difference in three out of five alpha-diversity measures estimated number of observed genera, Chao1 and ACE indices - demonstrating that catheterised urine displays smaller degree of richness and less variability than voided urine. Beta-diversity comparisons using different stringency levels (removed singletons and doubletons, 100 most abundant OTUs among all samples and the 3 most abundant taxa per sample, respectively Fig. $3 \mathrm{~A}, \mathrm{~B}$ and $\mathrm{C}$ ) for filtering the contingency table under the Bray-Curtis [28] distance resulted in random patterns, no clear grouping between the three urine sampling methods and no statistic significance when comparing the distance using PERMANOVA. Clear patterns of microbial community dissimilarities appeared only after filtering the contingency table using the PIME [26] method (Fig. 3D), i.e. removing noisy OTUs from the samples and keeping only the OTUs that are adding biological meaning to the group of samples (listed in Supplementary Table S3, Additional file 3). These OTUs were not necessarily the most abundant ones, their relative abundance ranging between $0.039 \%$ and $4.652 \%$. Some of them have not been associated with the human urinary tract so far (the genera Ferroglobus, Streptophyta, Cutibacterium, Kiritimatiella, Rhodococcus, Candidatus Scalindua and Methanopyrus). And while some of them may represent misalignment in the prokaryotic databases (such as the Archaea Ferroglobus and Methanopyrus), others might reflect real members of the male urinary microbiota.

PIME is one of the most recent machine learning approaches to identify relevant OTUs in microbiome analyses. It has been proved useful to classify even samples of patients with psychiatric disorders [29] using only intestinal microbiota data. Such separation can be seen at the non-metric multidimensional scaling (NMDS) plot of the beta-diversity analysis. The error to prevalence trade-off is supplied as Supplementary Table S4 (Additional file 4). Clear alpha-diversity differences and the beta-diversity pattern seen in the NMDS after the PIME prevalence filtering confirm previous observations that different sections of the lower urinary tract harbour a specific microbial community. Urine obtained by catheterisation also displays a less variable community structure, with its samples more similar to each other when considering the signature OTUs identified through the Random forest models (Fig. 3D).

The topic of a "core" urinary microbiome is an intriguing one and unlike in females where Lactobacillus, Prevotella and Gardnerella [2][30] seem to be the most commonly represented genera, there is a paucity of data on the dominant OTUs in MUM. While a study on patients from an STD clinic did not find clear evidence for a "core" microbiota in FCU samples [31], another study employed MSU samples of 11 healthy volunteers and suggested Streptococcus, Prevotella, Veillonella, Peptoniphilus, Campylobacter and Anaerococcus to represent the core healthy MUM [32]. The only work that tried to define core MUM based on catheterised urine of males reported Streptococcus to be the dominant genus in males [9]. According to our data, the most common dominant genera in catheterised urine specimens include Pseudomonas, Prevotella and Ferroglobus. While the frequent presence of Pseudomonas and Prevotella in urine seems biologically plausible, Ferroglobus spp. is an anaerobic, $\mathrm{Fe}^{2+}$-oxidizing archaeum isolated from a submarine hydrothermal system [33]. There seems to be a misalignment in the process of OTU identification here which illustrates one of the weak points of current urinary microbiota investigations: public databases are inadequate for this purpose as they lack urobiome-specific genomes [34]. 
Although the urinary microbiome is likely to incur some, yet undefined changes during lifetime, our data disprove the hypothesis postulated by one group that the genera Jonquetella, Parvimonas, Proteiniphilum and Saccharofermentans only dwell in bladders of people over 70 years old [35]. In our population, these OTUs were detected in 14 patients with a mean age of 66 years and some as young as 39 .

The discrepancy in the yield of microorganisms from voided and catheterised urine seen in NGS extends to the study of MUM by culture. We demonstrated that voided urine of asymptomatic men with a negative standard urine culture harbours living bacteria that can be cultured using EQUC. By contrast, catheterised urine from their urinary bladders only rarely yielded a positive EQUC result. Six different sets of culture conditions were employed by our EQUC protocol. Suppmentary table S2 illustrates their respective efficacies.

Hilt et al. were the first to introduce the concept of EQUC into the study of human urinary microbiome [6]. In their landmark study, $80 \%$ of catheterised female urine specimens reported as "no growth" at $10^{3}$ by the standard urine culture protocol yielded a positive result using EQUC. The most prevalent genera were Lactobacillus, Corynebacterium and Streptococcus. Another study of female urinary microbiota reported $89 \%$ positive EQUC results from catheterised urine samples of 75 women not reporting signs and symptoms of UTI; most of bacterial species were not detected by standard urine culture. Median of 3 isolates per sample (IQR 1-5) were detected and Streptococcus, Lactobacillus and Corynebacterium (in order of prevalence) were the most prevalent genera [3].

Several explanations can be given for the discrepancy between the yield of bacteria from catheterised urine in our and the abovementioned studies. Anatomical differences in the lower urinary tract of men and women seem the most obvious [36]: a long, twice curved male urethra as opposed to a straight, short and wide urethra in females; one that opens in direct proximity to the vaginal introit and not far from the anus. The vicinity of vaginal environment would also explain Lactobacillus being one of top-three bladder-dwellers in women but not in men. Another explanation for a higher detection rate of microorganisms by EQUC in the Hilt et al. and Price et al. studies [6], [37] might be less stringent enrolment criteria (no growth at $10^{3} \mathrm{CFU} / \mathrm{mL}$ and absence of UTI symptoms, respectively) compared to our study protocol. Lastly, minor modifications of our EQUC technique might have influenced the results.

In the only other work employing EQUC for the study of male urinary microbiome, Bajic et al. detected cultivable bacteria in $96 \%$ of voided urine specimens, a figure strikingly similar to our detection rate; among catheterised urine samples, $29 \%$ were positive in their study [10]. Because they did not perform standard urine culture, no comments on the pre-enrolment microbiological status of the study subjects can be made and, as noted earlier, all of our EQUC study participants preoperative urine samples were reported uniformly negative.

A subset of samples from 18 patients from our population were subjected to both EQUC and NGS in order to find out whether 16S rRNA gene sequences of the microorganisms cultured by EQUC are detectable by NGS. DNA sequences from 61 of 77 (79\%) EQUC-detected microorganisms were demonstrable by NGS; 
for 16 isolates, NGS failed to detect their DNA. This is a recognised phenomenon reported in two other studies combining EQUC and NGS for the detection of urinary microbiota [6], [38]. The culture-positive microorganisms were not necessarily the most abundant ones; on the contrary, mean relative abundance of the culture-proven microbes was $9.4 \%$ and varied widely from $0 \%$ to $93.2 \%$.

When it comes to the limitations of the current study, the following points should be recognized. The taxonomy assignment in high-throughput sequencing amplicon studies rely on a very small segment of the marker gene, considering the whole 16S rRNA gene contains approximately 1600 base pairs; we have used fragments of around $290 \mathrm{bp}$, therefore the identification of the taxa found can be misleading, as evidenced in the case of likely misalignment of Ferroglobus sequences (see above). Multiple copies of the 16S rRNA marker gene can influence the abundance of the taxa identified in the study and no reliable database to correct for this bias exists so far, therefore there might be orders of magnitude errors in the ranking of the genera present in a sample. Clustering sequences into OTUs has the limitation of grouping taxa that could behave differently and multiple copies of the marker gene could separate the same taxa into two different OTUs; moreover, two OTUs belonging to the same genera could grow differently in the same treatment, being different species or even different strains of the same genus. All of these biases become noise in the identification of patterns in the microbial composition of a sample. However, these flaws are inherent to microbiota investigations in general and this study is no exception. It ought to be noted that subjects of the study were recruited from patients of a urology department and not from a healthy population. This should not be a major issue since the study focuses on intra-individual variations of the microbiota. Finally, some suggest that DNA extraction protocols that employ bead beating for cell lysis give a better representation of bacterial community structure [39]. Our method of DNA extraction relied on a commercial kit that did not employ a mechanical cell disruption procedure. It should be noted, though, that in the context of the existing microbiome literature, bead-beating is far from being standard practice.

\section{Conclusion}

In the present comparative study of male urinary microbiome, we demonstrated that urine samples obtained by transurethral catheterisation under aseptic conditions lead to different results on subsequent NGS analysis compared to spontaneously voided (first-catch and mid-stream) urine samples. Using EQUC we corroborated previous evidence from female urinary microbiota studies regarding the detection of living microorganisms in samples of urine that are classified as "no growth" on standard urine culture. Catheterised urine is the most appropriate way to sample male bladder microbiota in future studies of urinary bladder pathological conditions and their relation to the urinary microbiota.

\section{Abbreviations}

CBA Columbia blood agar

EQUC extended quantitative urinary culture 
FCU first-catch urine

IQR inter-quartile range

MSU mid-stream urine

MUM male urinary microbiota

NGS next-generation sequencing

NMDS non-metric multidimensional scaling

OTU operational taxonomic unit

PCR polymerase chain reaction

PIME Prevalence Interval for Microbiome Evaluation

UTI urinary tract infection

\section{Declarations}

Ethics approval and consent to participate

The study was conducted in accordance with the Declaration of Helsinki after previous approval by the Ethics Committee of the Institute for Clinical and Experimental Medicine and Thomayer Hospital with Multi-center Competence under the number G-19-01 and informed consent was obtained from all all participants prior to enrollment.

Consent for publication

Not applicable.

Availability of data and material

Some of the raw data generated and/or analysed during this study are included in this published article as supplementary files. Larger datasets (16S rDNA gene sequences) are available in the NCBI SRA database under the accession number PRJNA744742 (http://www.ncbi.nlm.nih.gov/bioproject/744742).

Competing interests

The authors declare that they have no competing interests.

Funding 
Supported by MH CZ - DRO (Thomayer University Hospital - TUH, 00064190). The funding body had no role in the design of the study or interpretation of data.

Authors' contributions

Conceptualization: JH. Data curation and formal analysis: JH, DM, PC. Funding acquisition: JH, RZ. Investigation: JH, PC, VH. Methodology: JH, DM, PC. Project administration: JH. Visualization: DM, JH. Writing: JH, DM, PC. All authors read and approved the final manuscript.

Acknowledgements

The authors are grateful to Petra Tlaskalova for her support with Illumina sequencing.

\section{References}

1. Structure, function and diversity of the healthy human microbiome. Nature [Internet]. 2012;486:207-14. Available from: http://dx.doi.org/10.1038/nature11234

2. Siddiqui $\mathrm{H}$, Nederbragt AJ, Lagesen $\mathrm{K}$, Jeansson $\mathrm{SL}$, Jakobsen KS. Assessing diversity of the female urine microbiota by high throughput sequencing of 16S rDNA amplicons. BMC Microbiol. 2011; (11):1-12.

3. Price TK, Dune T, Hilt EE, Thomas-White KJ, Kliethermes S, Brincat C, et al. The Clinical Urine Culture: Enhanced Techniques Improve Detection of Clinically Relevant Microorganisms. J Clin Microbiol. 2016;54(5):1216-22.

4. Dong Q, Nelson DE, Toh E, Diao L, Gao X, Fortenberry JD, et al. The microbial communities in male first catch urine are highly similar to those in paired urethral swab specimens. PLoS One. $2011 ; 6(5): 1-5$.

5. Nelson DE, van der Pol B, Dong Q, Revanna K V., Fan B, Easwaran S, et al. Characteristic male urine microbiomes associate with asymptomatic sexually transmitted infection. PLoS One. 2010;5(11):1-7.

6. Hilt EE, McKinley K, Pearce MM, Rosenfeld AB, Zilliox MJ, Mueller ER, et al. Urine Is Not Sterile: Use of Enhanced Urine Culture Techniques To Detect Resident Bacterial Flora in the Adult Female Bladder. J Clin Microbiol. 2014;52(3):871-6.

7. Thomas-White K, Forster SC, Kumar N, Kuiken M Van, Putonti C, Stares MD, et al. Culturing of female bladder bacteria reveals an interconnected urogenital microbiota. Nat Commun [Internet]. 2018; (9):1557. Available from: http://dx.doi.org/10.1038/s41467-018-03968-5

8. Hourigan SK, Zhu W, Wong WSW, Clemency NC, Provenzano M, Vilboux T, et al. Studying the urine microbiome in superficial bladder cancer: samples obtained by midstream voiding versus 
cystoscopy. BMC Urol. 2020;20(5):1-8.

9. Pohl HG, Groah SL, Pérez-losada M, Ljungberg I, Sprague BM, Chandal N, et al. The Urine Microbiome of Healthy Men and Women Differs by Urine Collection Method. Int Neurourol J. 2020;24(1):41-51.

10. Bajic P, Kuiken ME van, Burge BK, Kirshenbaum EJ, Joyce CJ, Wolfe AJ, et al. Male Bladder Microbiome Relates to Lower Urinary Tract Symptoms. Eur Urol Focus [Internet]. 2020;15(6):376-82. Available from: https://doi.org/10.1016/j.euf.2018.08.001

11. Thomas-White KJ, Hilt EE, Fok C. Incontinence Medication Response Relates to the Female Urinary Microbiota. Int Urogynecol J. 2016;27(5):723-33.

12. Kramer H, Kuffel G, Thomas-White K, Wolfe AJ, Vellanki K, Leehey DJ, et al. Diversity of the midstream urine microbiome in adults with chronic kidney disease. Int Urol Nephrol [Internet]. 2018;50(6):1123-30. Available from: https://doi.org/10.1007/s11255-018-1860-7

13. Fouts D, Pieper R, Szpakowski S. Integrated next-generation sequencing of 16S rDNA and metaproteomics differentiate the healthy urine microbiome from asymptomatic bacteriuria in neuropathic bladder associated with spinal cord injury. J Transl Med. 2012;10(174).

14. Wu P, Zhang G, Zhao J, Chen J, Chen Y, Huang W, et al. Profiling the Urinary Microbiota in Male Patients With Bladder Cancer in China. Front Cell Infect Microbiol. 2018;8:167.

15. Pederzoli F, Ferrarese R, Amato V, Locatelli I, Alchera E, Luciano R, et al. Sex-specific Alterations in the Urinary and Tissue Microbiome in Therapy-naïve Urothelial Bladder Cancer Patients. Eur Urol Oncol. 2020;3(6):784-8.

16. Shoskes DA, Wang H, Polackwich AS, Tucky B, Altemus J, Eng C. Analysis of Gut Microbiome Reveals Significant Differences between Men with Chronic Prostatitis / Chronic Pelvic Pain Syndrome and Controls. J Urol [Internet]. 2016;196(2):435-41. Available from:

http://dx.doi.org/10.1016/j.juro.2016.02.2959

17. Siddiqui H, Lagesen K, Nederbragt AJ, Jeansson SL, Jakobsen KS. Alterations of microbiota in urine from women with interstitial cystitis. BMC Microbiol [Internet]. 2012;12:205. Available from: http://www.biomedcentral.com/1471-2180/12/205

18. Caporaso JG, Lauber CL, Walters WA, Berg-Lyons D, Lozupone CA, Turnbaugh PJ, et al. Global patterns of $16 \mathrm{~S}$ rRNA diversity at a depth of millions of sequences per sample. Proc Natl Acad Sci U S A. 2011 Mar;108 Suppl(Suppl 1):4516-22.

19. Vetrovsky T, Baldrian P, Morais D. SEED 2: a user-friendly platfomr for amplicon high-throughput sequencing data analyses. Bioinformatics. 2018;34(13):2292-4. 
20. Aronesty E. Comparison of Sequencing Utility Programs. Open Bioinforma J. 2013;7(1):1-8.

21. Edgar RC. UPARSE: highly accurate OTU sequences from microbial amplicon reads. Nat Methods. 2013 Oct;10(10):996-8.

22. Větrovský T, Baldrian P. Analysis of soil fungal communities by amplicon pyrosequencing: current approaches to data analysis and the introduction of the pipeline SEED. Biol Fertil Soils [Internet]. 2013;49(8):1027-37. Available from: https://doi.org/10.1007/s00374-013-0801-y

23. Cole JR, Wang Q, Fish JA, Chai B, McGarrell DM, Sun Y, et al. Ribosomal Database Project: Data and tools for high throughput rRNA analysis. Nucleic Acids Res. 2014;42(D1):633-42.

24. Oksanen AJ, Blanchet FG, Friendly M, Kindt R, Legendre P, Mcglinn D, et al. Package 'vegan .' Community Ecol Packag. 2017;5(December 2018).

25. McMurdie PJ, Holmes S. Phyloseq: An R Package for Reproducible Interactive Analysis and Graphics of Microbiome Census Data. PLoS One. 2013;8(4).

26. Roesch LFW, Dobbler PT, Pylro VS, Kolaczkowski B, Drew JC, Triplett EW. pime: A package for discovery of novel differences among microbial communities. Mol Ecol Resour. 2020 Mar;20(2):415-28.

27. Ihaka R, Gentleman R. R: A Language for Data Analysis and Graphics. J Comput Graph Stat [Internet]. 1996;5(3):299-314. Available from:

https://www.tandfonline.com/doi/abs/10.1080/10618600.1996.10474713

28. Chao A, Chazdon RL, Colwell RK, Shen T-J. A new statistical approach for assessing similarity of species composition with incidence and abundance data. Ecol Lett [Internet]. 2005;8(2):148-59. Available from: https://onlinelibrary.wiley.com/doi/abs/10.1111/j.1461-0248.2004.00707.x

29. Stevens BR, Roesch L, Thiago P, Russell JT, Pepine CJ, Holbert RC, et al. Depression phenotype identified by using single nucleotide exact amplicon sequence variants of the human gut microbiome. Mol Psychiatry. 2020 Jan;

30. Thomas-White KJ, Kliethermes S, Rickey L. Evaluation of the Urinary Microbiota of Women With Uncomplicated Stress Urinary Incontinence. Am J Obs Gynecol. 2017;216(1).

31. Frolund M, Wikstrom A, Lidbrink P, Al-soud WA, Larsen N, Ahrens P. The bacterial microbiota in first-void urine from men with and without idiopathic urethritis. PLoS One. 2018;13(7):e0201380.

32. Popović VB, Šitum M, Chow CT, Chan LS. The urinary microbiome associated with bladder cancer. Sci Rep. 2018;8:12157.

33. Hafenbradl D, Keller M, Dirmeier R, Rachel R, Rossnagel P, Burggraf S, et al. Ferroglobus placidus gen. nov., sp. nov., A novel hyperthermophilic archaeum that oxidizes $\mathrm{Fe} 2+$ at neutral $\mathrm{pH}$ under anoxic 
conditions. Arch Microbiol. 1996 Nov;166(5):308-14.

34. Wolfe AJ, Brubaker L. Urobiome updates: advances in urinary microbiome research. Nat Rev Urol. 2019;16(2):73-4.

35. Lewis DA, Brown R, Williams J, White P, Jacobson SK, Marchesi JR, et al. The human urinary microbiome; bacterial DNA in voided urine of asymptomatic adults. Front Cell Infect Microbiol [Internet]. 2013;3(August):1-14. Available from:

http://journal.frontiersin.org/article/10.3389/fcimb.2013.00041/abstract

36. Abelson B, Sun D, Que L, Nebel RA, Baker D, Popiel P, et al. Sex differences in lower urinary tract biology and physiology. Biol Sex Differ. 2018;9(1):1-13.

37. Price LB, Liu CM, Johnson KE, Aziz M, Lau MK, Bowers J, et al. The effects of circumcision on the penis microbiome. PLoS One. 2010;5(1):1-12.

38. Sathiananthamoorthy S, Malone-Lee J, Gill K, Tymon A, Nguyen TK, Gurung S, et al. Reassessment of Routine Midstream Culture in Diagnosis of Urinary Tract Infection. J Clin Microbiol. 2019;57(3):1-47.

39. Yuan S, Cohen DB, Ravel J, Abdo Z, Forney LJ. Evaluation of methods for the extraction and purification of DNA from the human microbiome. PLoS One. 2012;7(3).

\section{Figures}



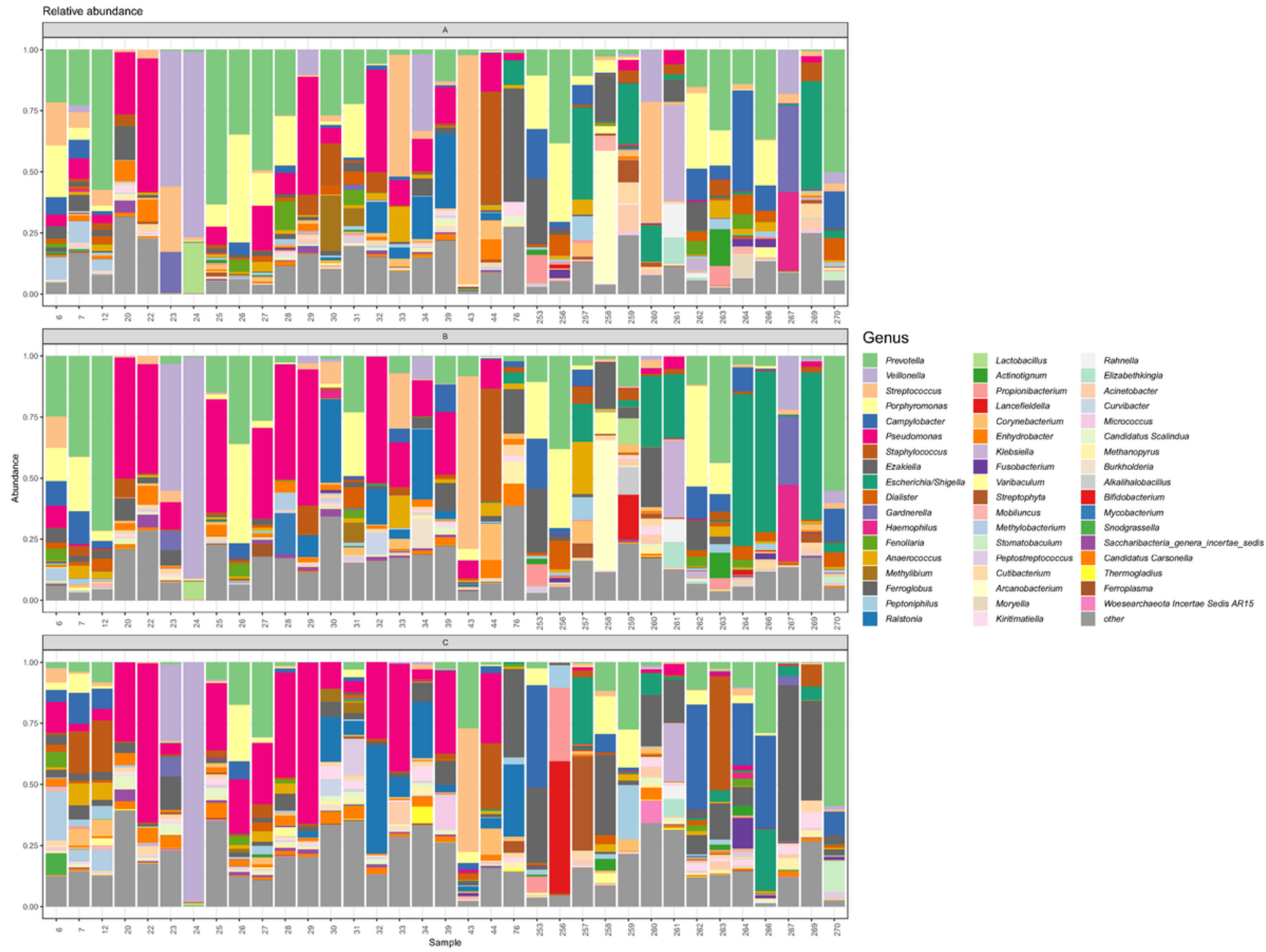

Figure 1

Barplots representing the relative abundance of OTUs in FCU (A), MSU (B) and catheterised (C) urine samples, respectively, at genus level. 

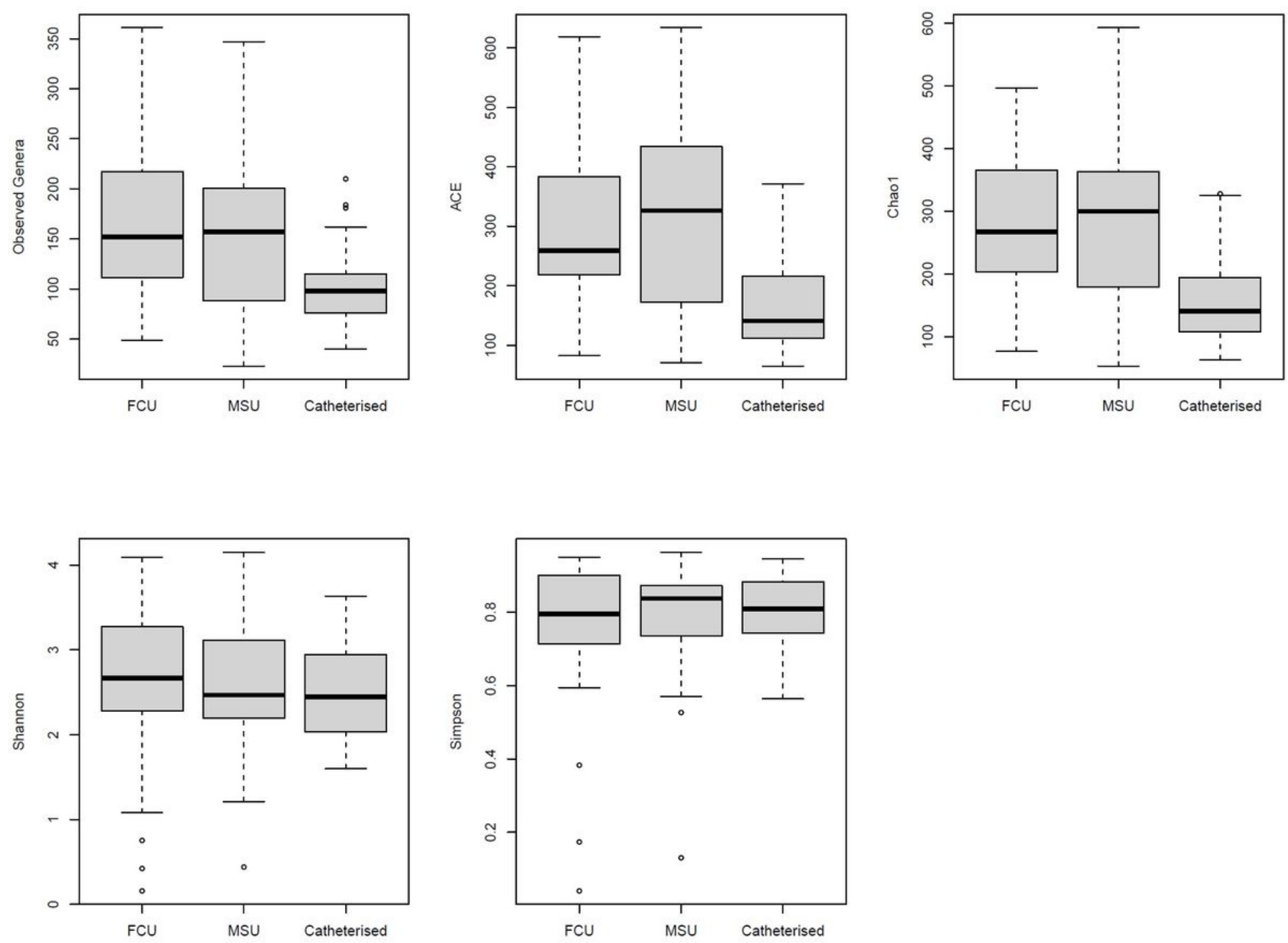

Figure 2

Boxplots comparing alpha-diversity measures for first-catch, mid-stream and catheterised urine. See also Table 2. 

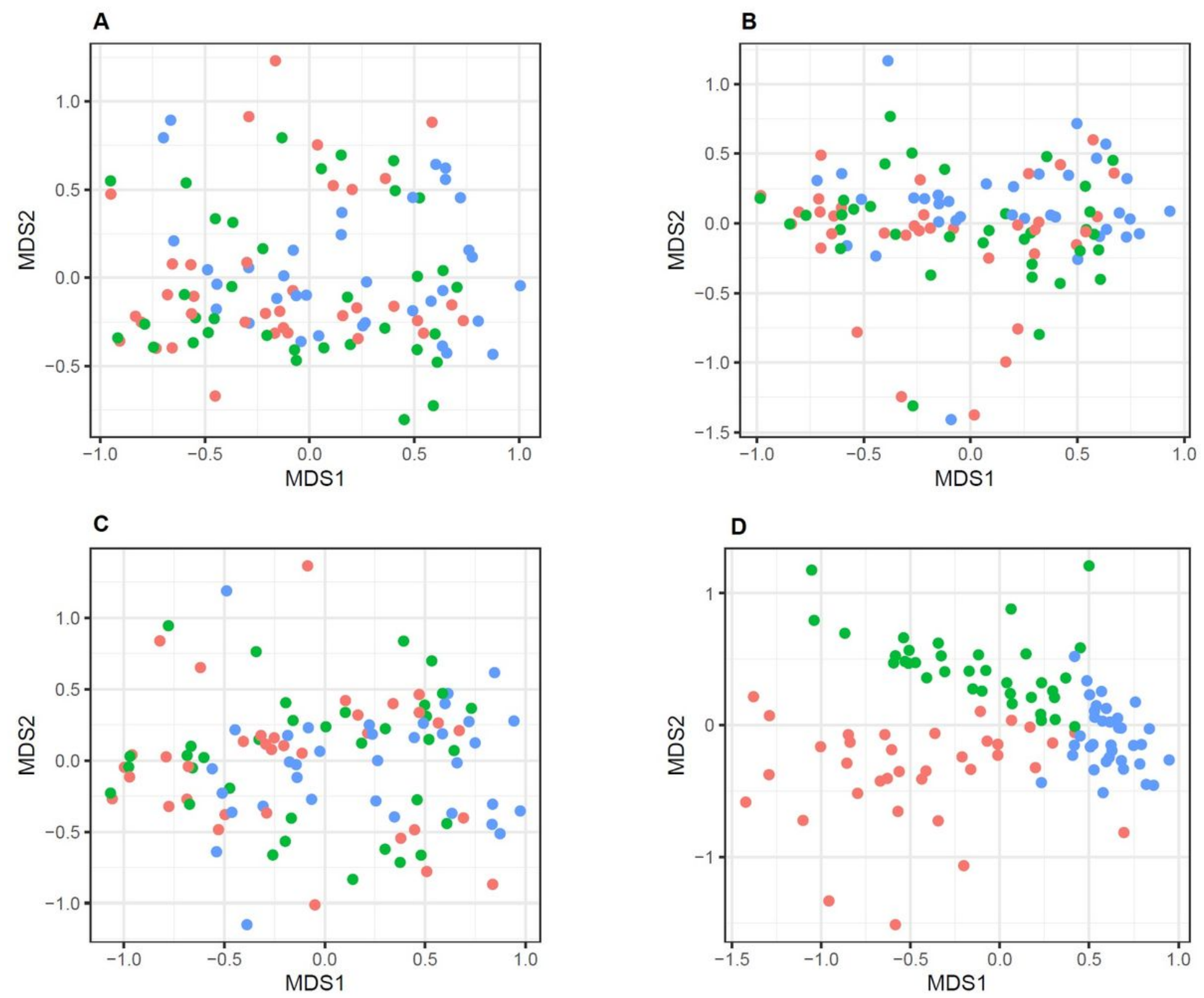

Figure 3

Bacterial community structures of urine depending on the way of sampling (red, first-catch urine; green, mid-stream urine; blue, catheterised urine), under 4 different filtering of OTUs (Figures 3A-D). NMDS ordination plots showing the dispersion of samples by their dissimilarity (Bray-Curtis index) after removal of erroneus sequences (Figure 3A), after keeping only the top 3 OTUs per sample (Figure 3B), after keeping the 100 most abundant OTUs in the whole dataset (Figure $3 \mathrm{C}$ ) and after filtering the most descriptive OTUs based on their prevalence using the PIME algorithm (Figure 3D).

\section{Supplementary Files}

This is a list of supplementary files associated with this preprint. Click to download. 
- AF1SupplementaryTable2EQUCresults.pdf

- AF2SupplementaryTable2EQUCperformance.pdf

- AF3SupplementarytableS3PIME.xIsx

- AF4SupplementarytableS4PIME.xlsx 